\title{
A Bertini-type theorem for free arithmetic linear series
}

\author{
Hideaki Ikoma
}

\begin{abstract}
In this paper, we prove a version of the arithmetic Bertini theorem asserting that there exists a strictly small and generically smooth section of a given arithmetically free graded arithmetic linear series.
\end{abstract}

\section{Introduction}

When we generalize results on arithmetic surfaces to those on higher-dimensional arithmetic varieties, it is sometimes very useful to cut the base scheme by a "good" global section $s$ of a given Hermitian line bundle and proceed to induction on dimension. To do this, we have in the context of Arakelov geometry the following result.

\section{FACT ([5, THEOREMS 4.2 AND 5.3])}

Let $\bar{A}$ be a $C^{\infty}$-Hermitian line bundle on a generically smooth projective arithmetic variety $X$, and let $x_{1}, \ldots, x_{q}$ be points (not necessarily closed) on $X$. Suppose that (i) $A$ is ample, (ii) $c_{1}(\bar{A})$ is positive definite, and (iii) $\mathrm{H}^{0}(X, m A$ ) has a $\mathbb{Z}$-basis consisting of sections with supremum norms less than 1 for every $m \gg 1$. Then there exist a sufficiently large integer $m \geq 1$ and a nonzero section $s \in \mathrm{H}^{0}(X, m A)$ such that

(1) $\operatorname{div}(s)_{\mathbb{Q}}$ is smooth over $\mathbb{Q}$,

(2) $s\left(x_{i}\right) \neq 0$ for every $i$, and

(3) $\|s\|_{\text {sup }}<1$.

For example, this technique plays essential roles in the proofs of the arithmetic Bogomolov-Gieseker inequality on high-dimensional arithmetic varieties (see [5]), of the arithmetic Hodge index theorem in codimension 1 (see [6], [10]), of the arithmetic Siu inequality of Yuan [9], and so on. A purpose of this paper is to give a simple elementary proof of the above fact and to strengthen it to the case of arithmetically free graded arithmetic linear series.

Kyoto Journal of Mathematics, Vol. 55, No. 3 (2015), 531-541

DOI 10.1215/21562261-3089037, (C) 2015 by Kyoto University

Received January 21, 2013. Accepted May 22, 2014.

2010 Mathematics Subject Classification: Primary 14G40; Secondary 11G50, 37P30.

Author's work supported by a research fellowship from the Japan Society for the Promotion of Science. 
Let $K$ be a number field. Let $X$ be a projective arithmetic variety that is geometrically irreducible over $\operatorname{Spec}\left(O_{K}\right)$, and let $L$ be an effective line bundle on $X$. A graded linear series belonging to $L$ is a subgraded $O_{K}$-algebra

$$
R_{\bullet}:=\bigoplus_{m \geq 0} R_{m} \subseteq \bigoplus_{m \geq 0} \mathrm{H}^{0}(X, m L)
$$

We consider norms $\|\cdot\|_{m}$ on $R_{m} \otimes_{\mathbb{Z}} \mathbb{R}$, and assume that the family of norms $\|\cdot\|_{\bullet}:=\left(\|\cdot\|_{m}\right)_{m \geq 0}$ is multiplicative, that is,

$$
\|s \otimes t\|_{m+n} \leq\|s\|_{m}\|t\|_{n}
$$

holds for every $s \in R_{m}$ and $t \in R_{n}$.

\section{THEOREM A}

Let $X$ be a generically smooth projective arithmetic variety, and let $A$ be an effective line bundle on $X$. We consider a graded linear series

$$
R_{\bullet}:=\bigoplus_{m \geq 0} R_{m}
$$

belonging to $A$ and a multiplicative norm $\|\cdot\|_{\bullet}$ on $R_{\bullet} \otimes_{\mathbb{Z}} \mathbb{R}$. Suppose the following conditions:

- $R_{1}$ is base point free,

- $R_{\bullet} \otimes_{\mathbb{Z}} \mathbb{Q}$ is generated by $R_{1}$ over $\mathbb{Q}$, and

- $\bigcap_{m \geq 1}\left\{x \in X_{\mathbb{Q}} \mid t(x)=0\right.$ for every $t \in R_{m}$ with $\left.\|t\|_{m}<1\right\}=\emptyset$.

Let $Y^{1}, \ldots, Y^{p}$ be smooth closed subvarieties of the complex manifold $X(\mathbb{C})$, and let $x_{1}, \ldots, x_{q}$ be points (not necessarily closed) on $X$. Then, for every sufficiently large integer $m \gg 1$, there exists a nonzero section $s \in R_{m}$ such that

(a) $\operatorname{div}\left(\left.s\right|_{Y^{1}}\right), \ldots, \operatorname{div}\left(\left.s\right|_{Y^{p}}\right)$ are all smooth,

(b) $s\left(x_{i}\right) \neq 0$ for every $i$, and

(c) $\|s\|_{m}<1$.

Let $\bar{L}$ be a continuous Hermitian line bundle on $X$, and let $\|\cdot\|_{\mathrm{sup}}^{(m)}$ be the supremum norm on $\mathrm{H}^{0}(X, m L) \otimes_{\mathbb{Z}} \mathbb{R}$. We define a $\mathbb{Z}$-submodule of $\mathrm{H}^{0}(X, m L)$ by

$$
\mathrm{F}^{0+}(X, m \bar{L}):=\left\langle s \in \mathrm{H}^{0}(X, m L) \mid\|s\|_{\text {sup }}^{(m)}<1\right\rangle_{\mathbb{Z}} .
$$

Then $\bigoplus_{m \geq 0} \mathrm{~F}^{0+}(X, m \bar{L})$ is a graded linear series belonging to $L$. We denote the stable base locus of $\bigoplus_{m \geq 0} \mathrm{~F}^{0+}(X, m \bar{L})$ by $\mathrm{SBs}^{0+}(\bar{L})$.

\section{COROLLARY B}

Let $X$ be a generically smooth projective arithmetic variety, and let $\bar{A}$ be a continuous Hermitian line bundle on $X$. Suppose that $\operatorname{SBs}(A)=\emptyset$ and $\operatorname{SBs}^{0+}(\bar{A}) \cap$ $X_{\mathbb{Q}}=\emptyset$. Let $Y^{1}, \ldots, Y^{p}$ be smooth closed subvarieties of the complex manifold $X(\mathbb{C})$, and let $x_{1}, \ldots, x_{q}$ be points (not necessarily closed) on $X$. Then there exist a sufficiently large integer $m \geq 1$ and a nonzero section $s \in \mathrm{H}^{0}(X, m A)$ such that 
(a) $\operatorname{div}\left(\left.s\right|_{Y^{1}}\right), \ldots, \operatorname{div}\left(\left.s\right|_{Y^{p}}\right)$ are all smooth,

(b) $s\left(x_{i}\right) \neq 0$ for every $i$, and

(c) $\|s\|_{\text {sup }}^{(m)}<1$.

\section{COROLLARY C}

Let $X$ be a generically smooth normal projective arithmetic variety, let $\bar{L}:=$ $\left(L,|\cdot|_{L}\right)$ be a continuous Hermitian line bundle on $X$, and let $x_{1}, \ldots, x_{q}$ be points (not necessarily closed) on $X \backslash \mathrm{SBs}^{0+}(\bar{L})$. If $\mathrm{SBs}^{0+}(\bar{L}) \subsetneq X$, then there exist a sufficiently large integer $m \geq 1$ and a nonzero section $s \in \mathrm{H}^{0}(X, m L)$ such that

(a) $\operatorname{div}(s)_{\mathbb{Q}}$ is smooth off $\mathrm{SBs}^{0+}(\bar{L})$,

(b) $s\left(x_{i}\right) \neq 0$ for every $i$, and

(c) $\|s\|_{\text {sup }}^{(m)}<1$.

Notation and conventions. Let $k$ denote a field, and let $\mathbb{P}^{n}:=\mathbb{P}\left(k^{n+1}\right)$ denote the projective space of one-dimensional quotients of $k^{n+1}$. Let $\mathrm{pr}_{2}: \mathbb{P}^{n} \times_{k} \mathbb{P}^{m} \rightarrow \mathbb{P}^{m}$ denote the second projection. We denote the natural coordinate variables of $\mathbb{P}^{n}$ (resp., of $\mathbb{P}^{m}$ ) by $X_{0}, \ldots, X_{n}$ (resp., by $Y_{0}, \ldots, Y_{m}$ ) or simply by $X_{\bullet}\left(\right.$ resp., by $Y_{\bullet}$ ).

Let $Y$ be a smooth variety over $k$. The singular locus of a morphism $\varphi: X \rightarrow$ $Y$ over $k$ is a Zariski-closed subset of $X$ defined as

$$
\operatorname{Sing}(\varphi):=\{x \in X \mid \varphi \text { is not smooth at } x\} .
$$

A projective arithmetic variety $X$ is a reduced irreducible scheme that is projective and flat over $\operatorname{Spec}(\mathbb{Z})$. We say that $X$ is generically smooth if $X_{\mathbb{Q}}:=$ $X \times_{\operatorname{Spec}(\mathbb{Z})} \operatorname{Spec}(\mathbb{Q}) \rightarrow \operatorname{Spec}(\mathbb{Q})$ is smooth

\section{Bertini's theorem with degree estimate}

In this section, we consider a geometric case. Let $X \subseteq \mathbb{P}^{n}$ be a projective variety over an algebraically closed field $k$ that is defined by a homogeneous prime ideal $I_{X} \subseteq k\left[X_{0}, \ldots, X_{n}\right]$, let $\mathcal{O}_{X}(1)$ be the hyperplane line bundle on $X$, and let

$$
\operatorname{deg} X:=\operatorname{deg}\left(c_{1}\left(\mathcal{O}_{X}(1)\right)^{\cdot \operatorname{dim} X}\right)
$$

be the degree of $X$ in $\mathbb{P}^{n}$. Let $k[X]:=k\left[X_{0}, \ldots, X_{n}\right] / I_{X}$ be the homogeneous coordinate ring of $X$, and let $k[X]_{l}$ be the homogeneous part of $k[X]$ of degree $l$. There exists a polynomial function $\varphi_{X}(l)$ such that $\operatorname{deg} \varphi_{X}=\operatorname{dim} X$, all coefficients are nonnegative, and

$$
\operatorname{dim}_{k} k[X]_{l} \leq \varphi_{X}(l)
$$

for all $l \geq 0$. Let $Z \subseteq X \times_{k} \mathbb{P}^{m}$ be a Zariski-closed subset defined by a system of polynomial equations:

$$
u_{1}\left(X_{\bullet} ; Y_{\bullet}\right)=0 \quad\left(\bmod I_{X}\right), \quad \ldots, \quad u_{h}\left(X_{\bullet} ; Y_{\bullet}\right)=0 \quad\left(\bmod I_{X}\right),
$$

where $u_{i} \in k\left[X_{0}, \ldots, X_{n} ; Y_{0}, \ldots, Y_{m}\right]$ has homogeneous degree $\operatorname{deg}_{X} u_{i}$ (resp., $\left.\operatorname{deg}_{Y_{\bullet}} u_{i}\right)$ in the set of variables $X_{\bullet}$ (resp., $\left.Y_{\bullet}\right)$. We recall the following fact from the elimination theory. 
LEMMA 1.1

Let $p:=\max _{i}\left\{\operatorname{deg}_{X_{\bullet}} u_{i}\right\}$, and let $q:=\max _{i}\left\{\operatorname{deg}_{Y_{\bullet}} u_{i}\right\}$. If the set-theoretic image $\operatorname{pr}_{2}(Z)$ does not coincide with $\mathbb{P}^{m}$, then $\mathrm{pr}_{2}(Z)$ is contained in a hypersurface of $\mathbb{P}^{m}$ defined by a single homogeneous polynomial of degree less than or equal to

$$
\varphi_{X}\left(\operatorname{deg} X \cdot p^{\operatorname{dim} X+1}\right) \cdot q .
$$

\section{Proof}

First, we can take a geometric point $y_{0, \bullet}=\left(y_{0,0}: \cdots: y_{0, m}\right) \in \mathbb{P}^{m} \backslash \operatorname{pr}_{2}(Z)$. By an effective Nullstellensatz (see [3, Corollary 1.4]), there exists a positive integer $\ell \leq \operatorname{deg} X \cdot p^{\operatorname{dim} X+1}$ such that

$$
\left(X_{0}, \ldots, X_{n}\right)^{\ell} \subseteq\left(u_{1}\left(X_{\bullet} ; y_{0, \bullet}\right), \ldots, u_{h}\left(X_{\bullet} ; y_{0, \bullet}\right)\right) \quad\left(\bmod I_{X}\right)
$$

Next, we consider the $k$-linear maps

$$
\begin{aligned}
T\left(y_{\bullet}\right): k[X]_{\ell-\operatorname{deg}_{X} u_{\bullet}} \oplus \cdots \oplus k[X]_{\ell-\operatorname{deg}_{X} u_{h}} & \rightarrow k[X]_{\ell}, \\
\left(f_{1}\left(X_{\bullet}\right), \ldots, f_{h}\left(X_{\bullet}\right)\right) & \mapsto \sum_{i} u_{i}\left(X_{\bullet} ; y_{\bullet}\right) f_{i}\left(X_{\bullet}\right)
\end{aligned}
$$

defined for $y_{\bullet}=\left(y_{0}: \cdots: y_{m}\right) \in \mathbb{P}^{m}$. By fixing a basis for the above $k$-vector spaces, we can represent $T\left(y_{\bullet}\right)$ by a matrix whose entries are homogeneous polynomials of $y$. of degree less than or equal to $q$. By the choice of $\ell$, we can see that there exists a certain $\operatorname{dim}_{k} k[X]_{\ell} \times \operatorname{dim}_{k} k[X]_{\ell}$-minor of the representation matrix of $T\left(y_{\bullet}\right)$ whose determinant is nonzero (see [8, Theorem 2.23]). Then the image $\operatorname{pr}_{2}(Z)$ is contained in the hypersurface defined by the nonzero determinant, which is homogeneous of degree less than or equal to $\left(\operatorname{dim}_{k} k[X]_{\ell}\right) \cdot q$. Since

$$
\operatorname{dim}_{k} k[X]_{\ell} \leq \varphi_{X}(\ell) \leq \varphi_{X}\left(\operatorname{deg} X \cdot p^{\operatorname{dim} X+1}\right),
$$

we have the result.

\section{REMARK 1.2}

For example, we consider the case where $X=\mathbb{P}^{n}$. Then $\operatorname{dim}_{k} k[X]_{l}=\left(\begin{array}{c}l+n \\ n\end{array}\right) \leq$ $(l+n)^{n} / n$ !. Thus, the bound in the above lemma becomes less than or equal to $\left(p^{n+1}+n\right)^{n} q / n$ !. Moreover, by applying the theory of resultants (see [8, page 35]) to $\mathrm{pr}_{2}: \mathbb{P}^{n} \times{ }_{k} \mathbb{A}^{m} \rightarrow \mathbb{A}^{m}$, one can obtain a weaker bound less than or equal to $(2 p)^{2^{n}-1} q+1$ in the above lemma (where the added 1 is for the hyperplane at infinity).

Let $A$ be an effective line bundle on $X$, and let $R_{0}$ be a subgraded ring of $\bigoplus_{m \geq 0} \mathrm{H}^{0}(X, m A)$ with Kodaira-Iitaka dimension $\kappa(R \cdot):=\operatorname{tr} \cdot \operatorname{deg}_{k} R \cdot-1$. Suppose that $R_{1}$ is base point free. Let $\phi_{m}: X \rightarrow \mathbb{P}\left(R_{m}\right)$ be a $k$-morphism associated to $R_{m}$, and set

$$
N_{m}:=\operatorname{dim}_{k} R_{m}-1
$$


for $m \geq 1$. We recall that the rational function field $k(X)$ of $X$ is given by

$$
k(X)=\left\{\begin{array}{l|l}
u\left(\bmod I_{X}\right) & \begin{array}{l}
u, v \in k\left[X_{0}, \ldots, X_{n}\right] \text { are homogeneous } \\
\text { of the same degree and } v \notin I_{X}
\end{array}
\end{array}\right\} .
$$

Given a nonzero section $e \in R_{1}$, we define the degree of a nonzero section $s \in$ $\mathrm{H}^{0}(X, m A)$ for $m \geq 1$ with respect to $e$ by

$$
\operatorname{deg}_{X_{\bullet}, e} s:=\min \left\{\begin{array}{l|l}
\operatorname{deg}_{X_{\bullet}} u=\operatorname{deg}_{X_{\bullet} v} & \begin{array}{l}
\operatorname{div} s=\left(u / v\left(\bmod I_{X}\right)\right)+m \operatorname{div} e, \\
u / v\left(\bmod I_{X}\right) \in k(X)^{\times}
\end{array}
\end{array}\right\} .
$$

(Compare the definition with Jelonek's in [3, Section 2].) Then, for any other nonzero section $s^{\prime} \in \mathrm{H}^{0}\left(X, m^{\prime} A\right)$, we have that

$$
\operatorname{deg}_{X_{\bullet}, e}\left(s \otimes s^{\prime}\right) \leq \operatorname{deg}_{X_{\bullet}, e} s+\operatorname{deg}_{X_{\bullet}, e} s^{\prime} .
$$

\section{THEOREM 1.3}

Let $X \subseteq \mathbb{P}^{n}$ be a smooth projective variety over $k$, and let $A$ be a line bundle on $X$. Let $R$. be a graded linear series belonging to A with Kodaira-Iitaka dimension $\kappa\left(R_{\bullet}\right)$. Suppose that the following three conditions are satisfied.

- $R_{1}$ is base point free.

- $R_{\text {. }}$ is generated by $R_{1}$.

- (i) $\operatorname{char}(k)=0$ or (ii) $\operatorname{char}(k) \neq 0$ and $\phi_{m}: X \rightarrow \mathbb{P}\left(R_{m}\right)$ is unramified for every $m \geq 1$.

Then one can find a polynomial function $P(m)$ and hypersurfaces $Z_{m} \subseteq \mathbb{P}\left(R_{m}^{\vee}\right)$ for $m=1,2, \ldots$ having the following two properties.

(a) $\operatorname{deg} P \leq \operatorname{dim} X(\operatorname{dim} X+1)\left(\kappa\left(R_{\bullet}\right)+1\right)$.

(b) For every $m \geq 1$, the hypersurface $Z_{m} \subseteq \mathbb{P}\left(R_{m}^{\vee}\right)$ contains the set

$$
\left\{H \in \mathbb{P}\left(R_{m}^{\vee}\right) \mid \phi_{m}(X) \subseteq H \text { or } \phi_{m}^{-1}(H) \text { is not smooth }\right\}
$$

and the homogeneous degree of $Z_{m}$ in $\mathbb{P}\left(R_{m}^{\vee}\right)$ is less than or equal to $P(m)$.

\section{REMARK 1.4}

Throughout this paper, we assume that the empty set $\emptyset$ is smooth, so that if $H \notin Z_{m}$, then $\phi_{m}^{-1}(H)$ is empty or smooth of pure dimension $\operatorname{dim} X-1$.

\section{Proof}

Let $I_{X} \subseteq k\left[X_{0}, \ldots, X_{n}\right]$ denote the homogeneous prime ideal defining $X$. We consider the universal hyperplane section

$$
W_{m}:=\left\{(x, H) \in X \times_{k} \mathbb{P}\left(R_{m}^{\vee}\right) \mid \phi_{m}(x) \in H\right\}
$$

endowed with the reduced induced scheme structure, and consider the restriction of the second projection $\operatorname{pr}_{2}: X \times_{k} \mathbb{P}\left(R_{m}^{\vee}\right) \rightarrow \mathbb{P}\left(R_{m}^{\vee}\right)$ to $W_{m}$, which we denote by

$$
\pi_{m}: W_{m} \rightarrow \mathbb{P}\left(R_{m}^{\vee}\right) .
$$


Note that $W_{m}$ is the inverse image of the canonical bilinear hypersurface in $\mathbb{P}\left(R_{m}\right) \times_{k} \mathbb{P}\left(R_{m}^{\vee}\right)$ via $\phi_{m} \times$ id $: X \times_{k} \mathbb{P}\left(R_{m}^{\vee}\right) \rightarrow \mathbb{P}\left(R_{m}\right) \times_{k} \mathbb{P}\left(R_{m}^{\vee}\right)$. Since the restriction of the first projection to $W_{m}, W_{m} \rightarrow X$, is surjective with fiber a projective space of dimension $N_{m}-1, W_{m}$ is irreducible. The set-theoretic image of the singular locus of $\pi_{m}$ is given by

$$
\pi_{m}\left(\operatorname{Sing}\left(\pi_{m}\right)\right)=\left\{H \in \mathbb{P}\left(R_{m}^{\vee}\right) \mid \phi_{m}(X) \subseteq H \text { or } \phi_{m}^{-1}(H) \text { is not smooth }\right\} .
$$

We fix a basis $e_{0}, \ldots, e_{N_{1}}$ for $R_{1}$. From now on, we explain a method to construct an equation $w_{0}$ that vanishes along $W_{m}$ from the section $e_{0}$. First, we set

$$
D_{1, e_{0}}:=\max _{1 \leq i \leq N_{1}}\left\{\operatorname{deg}_{X \bullet, e_{0}} e_{i}\right\}
$$

and take rational functions $u_{1}^{(1)} / v_{1}^{(1)}, \ldots, u_{N_{1}}^{(1)} / v_{N_{1}}^{(1)} \in k\left(X_{0}, \ldots, X_{n}\right)^{\times}$such that

$$
\operatorname{div} e_{i}=\left(\frac{u_{i}^{(1)}}{v_{i}^{(1)}}\left(\bmod I_{X}\right)\right)+\operatorname{div} e_{0} \quad \text { and } \quad \operatorname{deg}_{X \bullet} u_{i}^{(1)}=\operatorname{deg}_{X \bullet} v_{i}^{(1)} \leq D_{1, e_{0}}
$$

for $i=1, \ldots, N_{1}$. Next, for $m \geq 2$, we can choose sections $e_{1}^{(m)}, \ldots, e_{N_{m}}^{(m)} \in R_{m}$ such that

$$
e_{i}^{(m)} \in\left\{e_{0}^{\otimes \alpha_{0}} \otimes \cdots \otimes e_{N_{1}}^{\otimes \alpha_{N_{1}}} \mid \alpha_{0}+\cdots+\alpha_{N_{1}}=m\right\}
$$

and $e_{0}^{\otimes m}, e_{1}^{(m)}, \ldots, e_{N_{m}}^{(m)}$ form a basis for $R_{m}$. By identifying $\mathbb{P}\left(R_{m}^{\vee}\right)$ with $\mathbb{P}^{N_{m}}$ via the dual basis of $e_{0}^{\otimes m}, e_{1}^{(m)}, \ldots, e_{N_{m}}^{(m)}$, we can write $\phi_{m}: X \rightarrow \mathbb{P}\left(R_{m}^{\vee}\right)$ as

$$
\phi_{m}: X_{e_{0}} \rightarrow \mathbb{P}^{N_{m}}, \quad x \mapsto\left(1: \frac{u_{1}^{(m)}(x)}{v_{1}^{(m)}(x)}: \cdots: \frac{u_{N_{m}}^{(m)}(x)}{v_{N_{m}}^{(m)}(x)}\right)
$$

over $X_{e_{0}}:=\left\{x \in X \mid e_{0}(x) \neq 0\right\}$, where $u_{i}^{(m)} / v_{i}^{(m)} \in k\left(X_{0}, \ldots, X_{n}\right)^{\times}$satisfies

$$
\operatorname{div} e_{i}^{(m)}=\left(u_{i}^{(m)} / v_{i}^{(m)}\left(\bmod I_{X}\right)\right)+m \operatorname{div} e_{0}
$$

and

$$
\operatorname{deg}_{X \bullet} u_{i}^{(m)}=\operatorname{deg}_{X \bullet} v_{i}^{(m)} \leq D_{1, e_{0}} m
$$

We set

(1.6) $w_{0}:=v_{1}^{(m)} \cdots v_{N_{m}}^{(m)} Y_{0}+u_{1}^{(m)} v_{2}^{(m)} \cdots v_{N_{m}}^{(m)} Y_{1}+\cdots+v_{1}^{(m)} \cdots v_{N_{m}-1}^{(m)} u_{N_{m}}^{(m)} Y_{N_{m}}$, which is homogeneous in $X_{\bullet}$ (resp., in $Y_{\bullet}$ ) of degree less than or equal to $D_{1, e_{0}} m N_{m}$ (resp., 1). Then $w_{0}\left(\bmod I_{X}\right)$ vanishes along $W_{m}$ and defines $W_{m}$ in $X_{e_{0}} \times_{k} \mathbb{P}^{N_{m}}$.

By the same method, starting from $e_{j} \in R_{1}$, we can construct an equation

$$
\left.w_{j}=\sum \text { (homogeneous in } X_{\bullet} \text { of degree at most } D_{1, e_{j}} m N_{m}\right) \times\left(\text { linear in } Y_{\bullet}\right)
$$

that vanishes along $W_{m}$ and defines $W_{m}$ in $X_{e_{j}} \times_{k} \mathbb{P}^{N_{m}}$. Let $w_{N_{1}+1}, \ldots, w_{h} \in$ $k\left[X_{0}, \ldots, X_{n}\right]$ be homogeneous polynomials that generate $I_{X}$. Notice that the bihomogeneous ideal

$$
\left(w_{0}, \ldots, w_{N_{1}}, w_{N_{1}+1}, \ldots, w_{h}\right) \subseteq k\left[X_{0}, \ldots, X_{n} ; Y_{0}, \ldots, Y_{m}\right]
$$


may not be prime but the closed subscheme defined by $\left(w_{0}, \ldots, w_{h}\right)$ in $\mathbb{P}^{n} \times_{k} \mathbb{P}^{N_{m}}$ coincides with $W_{m}$.

Set

$$
D_{1}:=\max _{0 \leq i \leq N_{1}}\left\{D_{1, e_{i}}\right\}, \quad D_{2}:=\max _{N_{1}+1 \leq j \leq h}\left\{\operatorname{deg}_{X} w_{j}\right\}
$$

which does not depend on $m$. By the Euler rule together with the Jacobian criterion in the affine case, we conclude that the $\operatorname{singular}$ locus $\operatorname{Sing}\left(\pi_{m}\right) \subseteq X \times_{k}$ $\mathbb{P}\left(R_{m}^{\vee}\right)$ is defined by the determinants of certain $(n-\operatorname{dim} X+1) \times(n-\operatorname{dim} X+1)$ minors of the Jacobian matrix $\left(\frac{\partial w_{i}}{\partial X_{j}}\right)$, whose degrees in $X_{\bullet}$ (resp., in $Y_{\bullet}$ ) are all bounded from above by $\left(N_{1}+1\right)\left(D_{1} m N_{m}-1\right)+(n-\operatorname{dim} X)\left(D_{2}-1\right)$ (resp., by $\left.N_{1}+1\right)$. We choose a positive constant $D^{\prime}>0$ such that

$$
\left(N_{1}+1\right)\left(D_{1} m N_{m}-1\right)+(n-\operatorname{dim} X)\left(D_{2}-1\right) \leq D^{\prime} m^{\kappa\left(R_{\bullet}\right)+1}
$$

for all $m \geq 1$. Let $\varphi_{X}(l)$ be as in (1.1), and set

$$
P(m):=\varphi_{X}\left(\operatorname{deg} X\left(D^{\prime} m^{\kappa\left(R_{\bullet}\right)+1}\right)^{\operatorname{dim} X+1}\right) \cdot\left(N_{1}+1\right) .
$$

Then $\operatorname{deg} P=\operatorname{dim} X(\operatorname{dim} X+1)\left(\kappa\left(R_{\bullet}\right)+1\right)$. Since $\pi_{m}\left(\operatorname{Sing}\left(\pi_{m}\right)\right)$ is properly contained in $\mathbb{P}\left(R_{m}^{\vee}\right)$ due to Kleiman [4, Corollaries 5 and 12], we can apply Lemma 1.1 to this situation by setting

$$
p=D^{\prime} m^{\kappa\left(R_{\bullet}\right)+1} \quad \text { and } \quad q=N_{1}+1 .
$$

Then we conclude that there exists a hypersurface $Z_{m} \subseteq \mathbb{P}\left(R_{m}^{\vee}\right)$ having degree less than or equal to $P(m)$ and containing $\pi_{m}\left(\operatorname{Sing}\left(\pi_{m}\right)\right)$.

By applying Theorem 1.3 to the image of $R_{m}$ via $\mathrm{H}^{0}(X, m A) \rightarrow \mathrm{H}^{0}\left(Y,\left.m A\right|_{Y}\right)$, we have the following.

\section{COROLLARY 1.5}

Under the same assumptions as in Theorem 1.3, let $Y$ be a smooth closed subvariety of $X$, and let $y_{1}, \ldots, y_{q}$ be closed points on $X$. Then one can find a polynomial function $P(m)$ and hypersurfaces $Z_{m} \subseteq \mathbb{P}\left(R_{m}^{\vee}\right)$ for $m=1,2, \ldots$ having the following two properties.

(a) $\operatorname{deg} P \leq \operatorname{dim} Y(\operatorname{dim} Y+1)\left(\kappa\left(R_{\bullet}\right)+1\right)+q$.

(b) For every $m \geq 1$, the hypersurface $Z_{m} \subseteq \mathbb{P}\left(R_{m}^{\vee}\right)$ contains the set

$$
\left\{\begin{array}{l|l}
H \in \mathbb{P}\left(R_{m}^{\vee}\right) & \begin{array}{l}
\phi_{m}(Y) \subseteq H, \phi_{m}^{-1}(H) \cap Y \text { is not smooth, } \\
\text { or } H \text { contains one of } y_{1}, \ldots, y_{q}
\end{array}
\end{array}\right\},
$$

and the homogeneous degree of $Z_{m}$ in $\mathbb{P}\left(R_{m}^{\vee}\right)$ is less than or equal to $P(m)$.

\section{Proofs}

In this section, we turn to the arithmetic case and give proofs of Theorem A and Corollaries B and C. To prove Theorem A, we use Lemmas 2.1, 2.2, and 2.4. 
LEMMA 2.1 (COMBINATORIAL NULLSTELLENSATZ [5, LEMMA 5.2], [1, THEOREM 1.2])

Let $V$ be a finite-dimensional vector space over a field $k$, and let

$$
u: V \rightarrow k
$$

be a nonzero polynomial function with maximal total degree deg $u$. Let $e_{1}, \ldots, e_{N}$ be generators of $V$ over $k$, and let $S_{1}, \ldots, S_{N}$ be subsets of $k$. If $\operatorname{Card}\left(S_{j}\right) \geq$ $\operatorname{deg} u+1$ for every $j$, then there exist $a_{1} \in S_{1}, \ldots, a_{N} \in S_{N}$ such that

$$
u\left(a_{1} e_{1}+\cdots+a_{N} e_{N}\right) \neq 0 .
$$

\section{LEMMA 2.2}

Let $X$ be a projective arithmetic variety, let $A$ be a line bundle on $X$, and let $R_{\bullet}$ be a graded linear series belonging to $A$. Suppose that $R_{1}$ is base point free. Let $y_{1}, \ldots, y_{l} \in X$ be distinct closed points on $X$ such that $\operatorname{char}\left(k\left(y_{i}\right)\right) \neq 0$ for every $i$, and let $e_{1}^{(m)}, \ldots, e_{N_{m}}^{(m)} \in R_{m}$ be generators of the $\mathbb{Z}$-module $R_{m}$. Set $F:=\prod_{\exists i, p \mid \operatorname{char}\left(k\left(y_{i}\right)\right)} p$. Then, for every sufficiently large $m$, there exist integers $a_{1}, \ldots, a_{N_{m}}$ such that $0 \leq a_{j}<F$ for every $j$, and

$$
\left(a_{1}+F b_{1}\right) e_{1}^{(m)}\left(y_{i}\right)+\cdots+\left(a_{N_{m}}+F b_{N_{m}}\right) e_{N_{m}}^{(m)}\left(y_{i}\right) \neq 0
$$

for every integer $b_{1}, \ldots, b_{N_{m}}$ and for every $i$.

Proof

First, we need the following claim.

CLAIM 2.3

For every sufficiently large $m$, there exists an $s \in R_{m}$ such that $s\left(y_{i}\right) \neq 0$ for every $i$.

\section{Proof}

Let $\phi: X \rightarrow \mathbb{P}_{\mathbb{Z}}^{N_{1}}$ be the morphism associated to $R_{1}$ such that $\phi^{*} X_{j}=e_{j}^{(1)}$ for every $j$, and let $\mathcal{O}(1)$ be the hyperplane line bundle on $\mathbb{P}_{\mathbb{Z}}^{N_{1}}$. Then, for every sufficiently large $m$, the homomorphism

$$
\mathrm{H}^{0}\left(\mathbb{P}_{\mathbb{Z}}^{N_{1}}, \mathcal{O}(m)\right) \rightarrow \bigoplus_{i} \mathcal{O}(m)\left(\phi\left(y_{i}\right)\right)
$$

is surjective. Let $t \in \mathrm{H}^{0}\left(\mathbb{P}_{\mathbb{Z}}^{N_{1}}, \mathcal{O}(m)\right)$ be a section such that $t\left(\phi\left(y_{i}\right)\right) \neq 0$ for every $i$. Then $s:=\phi^{*} t$ has the desired property.

Next, let $s \in R_{m}$ as above. Since $F e_{j}^{(m)}\left(y_{i}\right)=0$ for every $i, j$, we have that

$$
(s+F t)\left(y_{i}\right)=s\left(y_{i}\right) \neq 0
$$

for every $t \in R_{m}$ and for every $i$. Thus we conclude the claim.

LEMMA 2.4 (ZHANG-MORIWAKI [7, THEOREM A AND COROLLARY B])

Under the same assumptions as in Theorem $A$, take an $m_{0} \gg 1$, and fix $e_{1}, \ldots$, 
$e_{N} \in R_{m_{0}}$ such that

$$
\left\{x \in X_{\mathbb{Q}} \mid e_{1}(x)=\cdots=e_{N}(x)=0\right\}=\emptyset
$$

and such that $\left\|e_{j}\right\|_{m_{0}}<1$ for every $j$. Then there exists a positive constant $C>0$ such that, for every sufficiently large $m$, one can find a $\mathbb{Z}$-basis $e_{1}^{(m)}, \ldots, e_{N_{m}}^{(m)}$ for $R_{m}$ such that

$$
\max _{i}\left\{\left\|e_{i}^{(m)}\right\|_{m}\right\} \leq C m^{(\operatorname{dim} X+2)(\operatorname{dim} X-1)}\left(\max _{j}\left\{\left\|e_{j}\right\|_{m_{0}}\right\}\right)^{m / m_{0}} .
$$

\section{Proof of Theorem $A$}

Let $r:=[K: \mathbb{Q}]$, and let $X(\mathbb{C})=X_{1} \cup \cdots \cup X_{r}$ be the decomposition into connected components. Let $R_{m, \alpha}$ be the image of $R_{m} \otimes_{\mathbb{Z}} \mathbb{C}$ via $\mathrm{H}^{0}(X, A) \otimes_{\mathbb{Z}} \mathbb{C} \rightarrow$ $\mathrm{H}^{0}\left(X_{\alpha},\left.A_{\mathbb{C}}\right|_{X_{\alpha}}\right)$, and let $\phi_{m, \alpha}: X_{\alpha} \rightarrow \mathbb{P}_{\mathbb{C}}^{M_{m}}$ be a morphism associated to $R_{m, \alpha}$, where we set $M_{m}:=\operatorname{rk}_{\mathbb{Z}} R_{m} / r$. By Lemma 2.4, there exist constants $C, Q$ with $C>0$ and $0<Q<1$ such that there exists a $\mathbb{Z}$-basis $e_{1}^{(m)}, \ldots, e_{r M_{m}}^{(m)}$ for $R_{m}$ consisting of the sections with supremum norms less than or equal to

$$
C m^{(\operatorname{dim} X+2)(\operatorname{dim} X-1)} Q^{m} \text {. }
$$

For each $Y^{j}$, there exists a unique component $X_{\alpha(j)}$ that contains $Y^{j}$. Suppose that $\operatorname{char}\left(x_{i}\right)=0$ for $i=1, \ldots, q_{1}$ and $\operatorname{char}\left(x_{i}\right) \neq 0$ for $i=q_{1}+1, \ldots, q=$ $q_{1}+q_{2}$, and let $y_{i}$ be a closed point in $\overline{\left\{x_{i}\right\}}$. By applying Corollary 1.5 to $X_{\alpha(j)}$, $Y^{j}, y_{1}, \ldots, y_{q_{1}}$, and $R_{\bullet}, \alpha(j)$, one can find a polynomial function $P_{j}(m)$ of degree less than or equal to $\operatorname{dim} Y^{j}\left(\operatorname{dim} Y^{j}-1\right)\left(\kappa\left(R_{\bullet}, \alpha(j)\right)+1\right)+q_{1}$ and hypersurfaces $Z_{m, j} \subseteq \mathbb{P}\left(R_{m, \alpha(j)}^{\vee}\right)$ defined by homogeneous polynomials $u_{m, j}$ of degree less than or equal to $P_{j}(m)$, respectively, such that $Z_{m, j}$ contains all the hyperplanes $H$ in $\mathbb{P}\left(R_{m, \alpha(j)}^{\vee}\right)$ such that $\phi_{m, \alpha(j)}\left(Y^{j}\right) \subseteq H, \phi_{m, \alpha(j)}^{-1}(H) \cap Y^{j}$ is not smooth, or $\phi_{m, \alpha(j)}^{-1}(H)$ contains one of $y_{1}, \ldots, y_{q_{1}}$. Set

$$
u_{m, \alpha}:=\prod_{\alpha(j)=\alpha} u_{m, j},
$$

and consider the homogeneous polynomial function

$$
u: R_{m} \otimes_{\mathbb{Z}} \mathbb{C} \stackrel{\sim}{\longrightarrow} \bigoplus_{\alpha=1}^{r} R_{m, \alpha} \stackrel{\prod_{\alpha} u_{m, \alpha}}{\longrightarrow} \mathbb{C}
$$

of degree less than or equal to

$$
P(m):=P_{1}(m)+\cdots+P_{p}(m) .
$$

Set $F:=\prod_{\exists i, q \mid \operatorname{char}\left(y_{i}\right)}^{q \text { a prime }} q$. Since $e_{1}^{(m)}, \ldots, e_{r M_{m}}^{(m)} \in R_{m}$ generate $R_{m} \otimes_{\mathbb{Z}} \mathbb{C}$ over $\mathbb{C}$, one can find integers $a_{1}, \ldots, a_{r M_{m}}$ and $b_{1}, \ldots, b_{r M_{m}}$ such that $0 \leq a_{i}<F$ for every $i, 0 \leq b_{j} \leq P(m)$ for every $j$, and

$$
u\left(\left(a_{1}+F b_{1}\right) e_{1}^{(m)}+\cdots+\left(a_{r M_{m}}+F b_{r M_{m}}\right) e_{r M_{m}}^{(m)}\right) \neq 0
$$


by use of Lemmas 2.2 and 2.1. Hence, for each $m \gg 1$, there exists a section $t_{m} \in R_{m}$ such that $\left.t_{m}\right|_{X_{\alpha}}$ is not contained in any of $Z_{m, j}$ and

$$
\left\|t_{m}\right\|_{m} \leq \text { CFrm }^{(\operatorname{dim} X+2)(\operatorname{dim} X-1)} M_{m}(1+P(m)) Q^{m} .
$$

Since the right-hand side tends to zero as $m \rightarrow \infty$, we conclude the proof.

Corollary B is a direct consequence of Theorem A.

\section{Proof of Corollary $C$}

We can take $a_{0} \gg 1$ such that $\operatorname{BsF}^{0+}\left(X, a_{0} \bar{L}\right)=\operatorname{SBs}^{0+}(\bar{L})$. Let $\mathfrak{b}^{0+}\left(a_{0} \bar{L}\right):=$ Image $\left(\mathrm{F}^{0+}\left(X, a_{0} \bar{L}\right) \otimes_{\mathbb{Z}}\left(-a_{0} L\right) \rightarrow \mathcal{O}_{X}\right)$, let $\mu: X^{\prime} \rightarrow X$ be a blowup such that $X^{\prime}$ is generically smooth and such that $\mu^{-1} \mathfrak{b}^{0+}\left(a_{0} \bar{L}\right) \cdot \mathcal{O}_{X^{\prime}}$ is Cartier, and let $E$ be an effective Cartier divisor on $X^{\prime}$ such that $\mathcal{O}_{X^{\prime}}(-E)=\mu^{-1} \mathfrak{b}^{0+}\left(a_{0} \bar{L}\right) \cdot \mathcal{O}_{X^{\prime}}$. We can assume that $\mu$ is isomorphic over $X \backslash \operatorname{SBs}^{0+}(\bar{L})$ (see [2]). Set $x_{i}^{\prime}:=\mu^{-1}\left(x_{i}\right) \in$ $X^{\prime} \backslash E$ for $i=1, \ldots, q$. Let $B:=\mathcal{O}_{X^{\prime}}(E)$, and let $1_{B}$ be the canonical section.

\section{LEMMA 2.5}

(a) We can endow $B$ with a continuous Hermitian metric $|\cdot|_{\bar{B}}$ such that

$$
\left|1_{B}\right|_{\bar{B}}(x)=\max _{\substack{e \in \mathrm{H}^{0}\left(X, a_{0} L\right) \\ 0<\|e\|_{\text {sup }}^{(a 0)}<1}}\left\{\frac{|e|_{a_{0} \bar{L}}(\mu(x))}{\|e\|_{\text {sup }}^{\left(a_{0}\right)}}\right\} \leq 1
$$

for all $x \in X^{\prime}(\mathbb{C})$.

(b) We set $\bar{B}:=\left(B,|\cdot|_{\bar{B}}\right)$ and $\bar{A}:=a_{0} \mu^{*} \bar{L}-\bar{B}$. Then $\bar{A}$ is a continuous Hermitian line bundle on $X^{\prime}$ such that

$$
\operatorname{BsF}^{0+}\left(X^{\prime}, \bar{A}\right)=\emptyset \quad \text { and } \quad c_{1}(\bar{A}) \geq 0
$$

as a current.

Proof

Set $\left\{e \in \mathrm{H}^{0}\left(X, a_{0} L\right) \backslash\{0\} \mid\|e\|_{\text {sup }}^{\left(a_{0}\right)}<1\right\}=\left\{e_{1}, \ldots, e_{N}\right\}$.

(a) We choose an open covering $\left\{U_{\nu}\right\}$ of $X^{\prime}(\mathbb{C})$ such that $\left.a_{0} \mu^{*} L_{\mathbb{C}}\right|_{U_{\nu}}$ is trivial with local frame $\eta_{\nu}$, and $E_{\mathbb{C}} \cap U_{\nu}$ is defined by a local equation $g_{\nu}$. Then we can write $\mu^{*} e_{j}=f_{j, \nu} \cdot g_{\nu} \cdot \eta_{\nu}$ on $U_{\nu}$, where $f_{1, \nu}, \ldots, f_{N, \nu}$ are holomorphic functions on $U_{\nu}$ satisfying $\left\{x \in U_{\nu} \mid f_{1, \nu}(x)=\cdots=f_{N, \nu}(x)=0\right\}=\emptyset$. Since

$$
\max _{j}\left\{\frac{\left|e_{j}\right|_{a_{0} \bar{L}}(\mu(x))}{\left\|e_{j}\right\|_{\text {sup }}^{\left(a_{0}\right)}}\right\}=\max _{j}\left\{\frac{\left|f_{j, \nu}(x)\right|}{\left\|e_{j}\right\|_{\text {sup }}^{\left(a_{0}\right)}}\right\} \cdot\left|\eta_{\nu}\right|_{a_{0} \mu^{*} \bar{L}}(x) \cdot\left|g_{\nu}(x)\right|
$$

on $x \in U_{\nu}$, we have (a).

(b) For each $x_{0} \in X^{\prime}(\mathbb{C})$, we take indices $\nu$ and $j_{0}$ such that $x_{0} \in U_{\nu}$ and $f_{j_{0}, \nu}\left(x_{0}\right) \neq 0$. Let $\varepsilon_{j}$ be the section of $A$ such that $\mu^{*} e_{j}=\varepsilon_{j} \otimes 1_{B}$, and set $h_{j, \nu}:=$ $f_{j, \nu} / f_{j_{0}, \nu}$. Then

$$
-\log \left|\varepsilon_{j_{0}}\right| \frac{2}{A}(x)=\max _{j}\left\{\log \left|h_{j, \nu}(x)\right|^{2}-\log \left(\left\|e_{j}\right\|_{\text {sup }}^{\left(a_{0}\right)}\right)^{2}\right\}
$$

is plurisubharmonic near $x_{0}$. 
We claim that $\left\|\varepsilon_{j}\right\|_{\text {sup }}=\left\|e_{j}\right\|_{\text {sup }}^{\left(a_{0}\right)}$, so that $\varepsilon_{j} \in \mathrm{F}^{0+}\left(X^{\prime}, \bar{A}\right)$. The inequality $\left\|\varepsilon_{j}\right\|_{\text {sup }} \geq\left\|e_{j}\right\|_{\text {sup }}^{\left(a_{0}\right)}$ is clear. Since

$$
\left|\varepsilon_{j}\right|_{\bar{A}}(x)=\left|e_{j}\right|_{a_{0} \bar{L}}(\mu(x)) \cdot \min _{i}\left\{\frac{\left\|e_{i}\right\|_{\text {sup }}^{\left(a_{0}\right)}}{\left|e_{i}\right|_{a_{0} \bar{L}}(\mu(x))}\right\} \leq\left\|e_{j}\right\|_{\text {sup }}^{\left(a_{0}\right)}
$$

for all $x \in\left(X^{\prime} \backslash E\right)(\mathbb{C})$, we have $\left\|\varepsilon_{j}\right\|_{\text {sup }}=\left\|e_{j}\right\|_{\text {sup }}^{\left(a_{0}\right)}$. This means that $\operatorname{BsF}^{0+}\left(X^{\prime}\right.$, $\bar{A})=\emptyset$.

We apply Corollary B to $\bar{A}$, and we can find an $m \gg 1$ and a $\sigma \in \mathrm{H}^{0}\left(X^{\prime}, m A\right)$ such that $\operatorname{div}(\sigma)_{\mathbb{Q}}$ is smooth, $\sigma\left(x_{i}^{\prime}\right) \neq 0$ for every $i$, and $\|\sigma\|_{\text {sup }}<1$. Since $X$ is normal, there exists an $s \in \mathrm{H}^{0}\left(X, m a_{0} L\right)$ such that $\mu^{*} s=\sigma \otimes 1_{B}^{\otimes m}$. Since $\mu$ is isomorphic over $X \backslash \operatorname{SBs}^{0+}(\bar{L}), s$ has the desired properties.

\section{References}

[1] N. Alon, Combinatorial Nullstellensatz, Combin. Probab. Comput. 8 (1999), 7-29. MR 1684621. DOI 10.1017/S0963548398003411.

[2] H. Hironaka, Resolution of singularities of an algebraic variety over a field of characteristic zero, I, Ann. of Math. (2) 79 (1964), 109-203; II, 205-326. MR 0199184.

[3] Z. Jelonek, On the effective Nullstellensatz, Invent. Math. 162 (2005), 1-17. MR 2198324. DOI 10.1007/s00222-004-0434-8.

[4] S. L. Kleiman, The transversality of a general translate, Compos. Math. 28 (1974), 287-297. MR 0360616.

[5] A. Moriwaki, Arithmetic Bogomolov-Gieseker's inequality, Amer. J. Math. 117 (1995), 1325-1347. MR 1350599. DOI 10.2307/2374978.

[6] Hodge index theorem for arithmetic cycles of codimension one, Math. Res. Lett. 3 (1996), 173-183. MR 1386838. DOI 10.4310/MRL.1996.v3.n2.a4.

[7]_ Free basis consisting of strictly small sections, Int. Math. Res. Not. IMRN 2011, no. 6, 1245-1267. MR 2806505. DOI 10.1093/imrn/rnq116.

[8] D. Mumford, Algebraic Geometry, I: Complex Projective Varieties, Grundlehren Math. Wiss. 221, Springer, Berlin, 1976. MR 0453732.

[9] X. Yuan, Big line bundles over arithmetic varieties, Invent. Math. 173 (2008), 603-649. MR 2425137. DOI 10.1007/s00222-008-0127-9.

[10] X. Yuan and S.-W. Zhang, The arithmetic Hodge index theorem for adelic line bundles, I, preprint, arXiv:1304.3538v1 [math.NT].

Graduate School of Mathematical Sciences, University of Tokyo, Tokyo, Japan;

ikoma@ms.u-tokyo.ac.jp 